\title{
Pan-Sharpening via Coupled Unitary Dictionary Learning
}

\author{
Shumiao Chen ${ }^{1(\bowtie)}$, Liang Xiao ${ }^{1,2}$, Zhihui $\mathrm{Wei}^{1}$, and Wei Huang ${ }^{1}$ \\ ${ }^{1}$ School of Computer Science and Engineering, \\ Nanjing University of Science and Technology, \\ Xiaolingwei Street 200, Nanjing 210094, Jiangsu, China \\ chenshumiao@126.com, \\ \{xiaoliang, gswei\}@mail.njust.edu.cn, hnhw235@163.com \\ ${ }^{2}$ Key Lab of Intelligent Perception and Systems for High-Dimensional \\ Information of Ministry of Education, \\ Xiaolingwei Street 200, Nanjing 210094, Jiangsu, China
}

\begin{abstract}
In this paper, we propose a new pan-sharpening method by coupled unitary dictionary learning and clustered sparse representation. First, we randomly sample image patch pairs from the training images exclude the smooth patches, and divide these patch pairs into different groups by K-means clustering. Then, we learn sub-dictionaries offline from corresponding group patch pairs. Particularly, we use the principal component analysis (PCA) technique to learn sub-dictionaries. For a given LR MS patch, we adaptively select one sub-dictionary to reconstruct the HR MS patch online. Experiments show that the proposed method produces images with higher spectral resolution while maintaining the high-quality spatial resolution and gives better visual perception compared with the conventional methods.
\end{abstract}

Keywords: Pan-sharpening · Sparse representation · Sub-dictionaries learning $\cdot$ K-means clustering

\section{Introduction}

Many optical Earth observation satellites, such as QuickBird, Worldview2, and IKONOS, provide two types of images: high resolution panchromatic images (PAN) and low resolution multispectral (MS) images. The multispectral image lacks high spatial quality and the panchromatic image has low spectral quality. However, there are a number of applications in remote sensing that require images of both high spatial and high spectral resolutions, such as object detection, land-cover classification, map updating [1]. The fusion of PAN and MS images is called "pan-sharpening". It provides a solution to this by fusing a high spatial resolution MS image from the input LR MS image and the HR PAN image.

\subsection{Related Work}

Over the years, a large collection of pan-sharpening methods has been proposed, which usually consider physics of the remote sensing process and make some assumptions on 
the original PAN and MS images. These fusion methods can be classified into three categories. First, projection-substitution methods assume that the PAN is equivalent to the structural component of the MS images when projected the MS images into a new space, for example, Intensity-Hue-Saturation Technique (IHS) [2] and its modifications such as the adaptive IHS (AIHS) [3], Principal Components Analysis (PCA) [4], Brovey transform [5]. Second, spatial details injection methods assume that the missing spatial information in the MS images can be obtained from the high frequencies of the PAN, such as wavelet-based fusion [6]. Third, the inverse-problem-based methods are used to restore the original high-resolution MS images from its degraded versions, i.e., the HR PAN and LR MS images.

Recently, Li and Zhu et al. proposed a novel pan-sharpening method using a compressed sensing technique and sparse representation [7, 8]. A critical issue in sparse representation modeling is the determination of dictionary. $\mathrm{Li}$ and $\mathrm{Zhu}$ generated the dictionary by randomly sampling or extracting all raw patches from the training image directly. In addition, analytically designed dictionaries, such as DCT, wavelet, curvelet, and contourlets, share the advantages of fast implementation. However, they lack the adaptivity to image local structures. There has been much effort in learning dictionaries from example image patches, such as K-SVD [9], leading to state-of-the-art results. These dictionary learning methods aim at learning a universal and over-complete dictionary to represent various image structures. However, the image contents can vary significantly across images. Sparse decomposition over a highly redundant dictionary is potentially unstable and tends to generate visual artifacts $[10,11]$. One may argue that a well-learned over-complete dictionary can sparsely code all of the possible image structures; nonetheless, for each given image patch, such a "universal" dictionary is neither optimal nor efficient because many atoms in the dictionary are irrelevant to the given local patch. These irrelevant atoms will not only reduce the computational efficiency in sparse coding but also reduce the representation accuracy.

\subsection{Our Contributions}

In this paper, we propose an adaptive sparse domain selection scheme for sparse representation. A set of compact sub-dictionaries is learned from the PAN image patches, which are clustered into many clusters. Since each cluster consists of many patches with similar patterns, a compact sub-dictionary can be learned for each cluster. In particular, we use the PCA to learn each sub-dictionary for simplicity. For a LR MS image patch to be coded, the best sub-dictionary that is most relevant to the given patch is selected. Thus, the given patch can be better represented by the adaptively selected sub-dictionary. Therefore, the whole image can be more accurately reconstructed than using a universal dictionary, which will be validated by our experiments.

The rest of this paper is organized into four sections. Section 2 presents the proposed method. Numerical experiments and discussions are presented in Sect. 3. Conclusions are given in Sect. 4. 


\section{Proposed Work}

Pan-sharpening requires a low-resolution multispectral (LR MS) image $\mathbf{Y}$ with $\mathrm{N}$ channels and a high-resolution panchromatic(HR PAN) image $\mathbf{X}_{h}$ and aims at increasing the spatial resolution of $\mathbf{Y}$ while keeping its spectral information, i.e., generating an HR MS image $\mathbf{X}$ utilizing both $\mathbf{Y}$ and $\mathbf{X}_{h}$ as inputs. While the SparseFI algorithm in Ref. [8] has achieved a very good pan-sharpening result compared with other conventional methods, there is still much room to improve this algorithm, especially in the dictionary training stage. In order to exploit the group similarity among image patch pairs, we propose coupled dictionary learning and clustered sparse representation method to improve the quality of the recovered HR MS image. The proposed method consists of three main steps: (1) coupled unitary sub-dictionaries learning; (2) sparse coefficients estimation; and (3) HR multispectral image reconstruction (see Fig. 1).

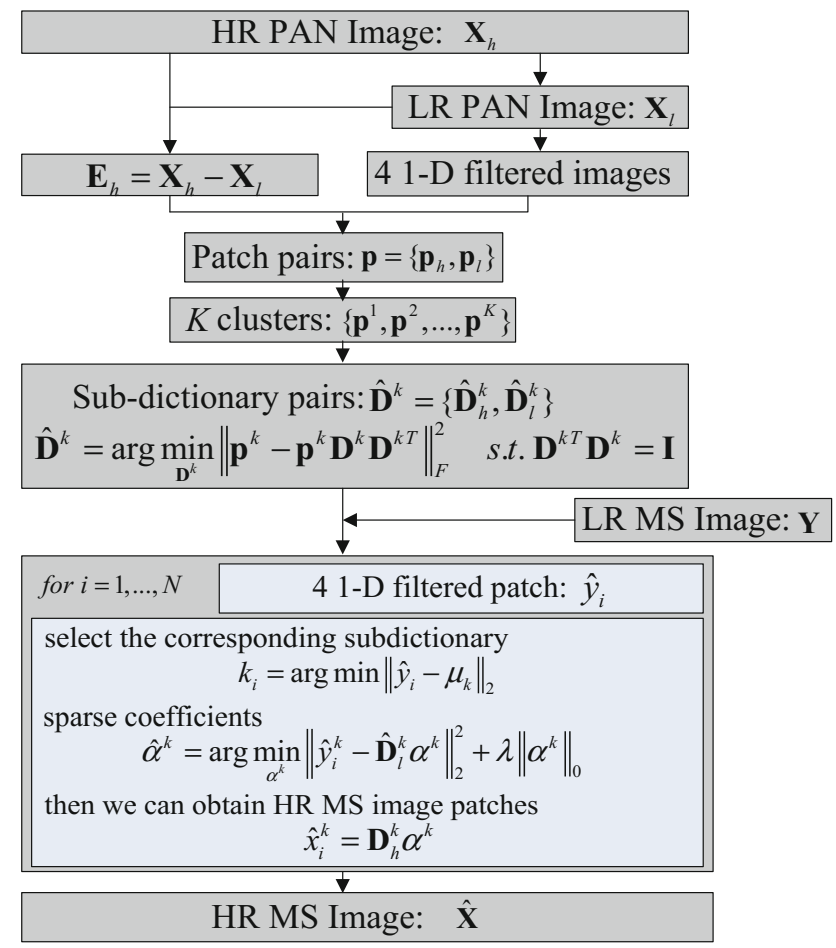

Fig. 1. Flow chart of the proposed method.

\subsection{Coupled Unitary Sub-dictionaries Learning}

The HR PAN image $\mathbf{X}_{h}$ is low-pass filtered and downsampled by a factor such that it has a final point spread function similar to a sampling grid identical to the multispectral channels, and then scaled-up by a bicubic interpolation operator that fills in the missing 
pixels to generate LR PAN image denoted by $\mathbf{X}_{l}$. Thus, $\mathbf{X}_{h}$ and $\mathbf{X}_{l}$ have the same size so that can avoid the complexity caused by different size.

In order to learn a series of sub-dictionaries to code the various local image structures, we need to first construct a dataset of HR and LR image patches for training. The training HR image patches $\mathbf{p}_{h}$ are extracted from the difference image $\mathbf{E}_{h}=\mathbf{X}_{h}-\mathbf{X}_{l}$, since we desire to focus the training on characterizing the relation between the LR patches and the edges and texture content within the corresponding HR ones [12]. In many literatures, people have suggested extracting different features for the LR image patch in order to boost the prediction accuracy. Freeman et al. [13] used a high-pass filter to extract the edge information from the LR input patches as the feature. Sun et al. [14] used a set of Gaussian derivative filters to extract the contours in the LR patches. Yang et al. [15] used the first-order and second-order gradients of the patches as the representation. In this paper, we also use the first-order and second-order derivatives as the feature for the LR patch due to its simplicity and effectiveness. It allows us to focus on the edges and structures of image patches without taking into account the pixel intensities. It helps to increase the accuracy of clustering. Here, the four 1-D filters used to extract the derivatives are:

$$
\begin{array}{cc}
f_{1}=[-1,0,1], & f_{2}=f_{1}^{T} \\
f_{3}=[1,0,-2,0,1], & f_{4}=f_{3}^{T}
\end{array}
$$

where the superscript " $T$ " means transpose.

We apply the four filters to the LR PAN image $\mathbf{X}_{l}$. Then we can get four gradient maps at each location and concatenate them to be the feature vector. The corresponding LR image patches $\mathbf{p}_{l}$ are extracted from the same locations in the filtered LR images. Therefore, the feature representation for each low-resolution image patch also encodes its neighboring information, which is beneficial for promoting compatibility among adjacent patches in the final fusion HR MS image.

In addition, we exclude the smooth patches from $\mathbf{p}_{h}$ and $\mathbf{p}_{l}$ and guarantee that only the meaningful patches with a certain amount of edge structures are involved in dictionary learning. We view the patch is smooth once its intensity variance is smaller than a predefined threshold $\Delta$.

Thus, local patches are extracted to form the data set $\mathbf{p}=\left\{\mathbf{p}_{h}, \mathbf{p}_{l}\right\}$. For coupled dictionary learning, we concatenate the HR patches $\mathbf{p}_{h}$ and LR patches $\mathbf{p}_{l}$ into one single vector via normalizing both feature vectors independently in order to balance their contributions. Clustered sparse representation partitions the dataset $\mathbf{p}$ into $\mathrm{K}$ clusters $\left\{\mathbf{P}^{1}, \mathbf{P}^{2}, \ldots, \mathbf{P}^{K}\right\}$ and denotes by $\mu_{k}$ the centroid of cluster $\mathbf{p}^{k}=\left\{\mathbf{p}_{h}^{k}, \mathbf{p}_{l}^{k}\right\}$ with K-means algorithm for simplicity. Then we can learn a sub-dictionary pair $\mathbf{D}^{k}=$ $\left\{\mathbf{D}_{h}^{k}, \mathbf{D}_{l}^{k}\right\}$ from each $\mathbf{p}^{k}$. Here, we use the feature of LR patches $\mathbf{p}_{l}$ regardless of HR patches $\mathbf{p}_{h}$ for clustering, since the LR patches are more reliable than the HR patches. In order to compute the sparse coefficients conveniently in stage two, we apply PCA to learn the sub-dictionary pair $\left\{\mathbf{D}_{h}^{k}, \mathbf{D}_{l}^{k}\right\}$ from each image patch pair $\left\{\mathbf{p}_{h}^{k}, \mathbf{p}_{l}^{k}\right\}$. PCA is a classical signal de-correlation and dimensionality reduction technique that is widely used in pattern recognition and statistical signal processing. In many literatures, PCA has been successfully used in spatially adaptive image denoising and super-resolution. 
The purpose of PCA is to find an orthogonal transformation matrix minimizing the error of reconstruction. So, the learning model of each sub-dictionary can be mathematically written as follows:

$$
\begin{cases}\hat{\mathbf{D}}_{h}^{k}=\underset{\mathbf{D}_{h}^{k}}{\arg \min _{h}\left\|\mathbf{p}_{h}^{k}-\mathbf{p}_{h}^{k} \mathbf{D}_{h}^{k} \mathbf{D}_{h}^{k T}\right\|_{F}^{2}} \quad \text { s.t. } \mathbf{D}_{h}^{k T} \mathbf{D}_{h}^{k}=\mathbf{I} \\ \hat{\mathbf{D}}_{l}^{k}=\underset{\mathbf{D}_{l}^{k}}{\arg \min }\left\|\mathbf{p}_{l}^{k}-\mathbf{p}_{l}^{k} \mathbf{D}_{l}^{k} \mathbf{D}_{l}^{k T}\right\|_{F}^{2} & \text { s.t. } \mathbf{D}_{l}^{k T} \mathbf{D}_{l}^{k}=\mathbf{I}\end{cases}
$$

where $\mathbf{p}_{h}^{k}$ denotes the $k$-th cluster of HR patches $\mathbf{p}_{h} ; \mathbf{p}_{l}^{k}$ denotes the $k$-th cluster of LR patches $\mathbf{p}_{l}$. Equation (2) can be rewritten as follows:

$$
\begin{gathered}
\hat{\mathbf{D}}^{k}=\underset{\mathbf{D}^{k}}{\arg \min }\left\|\mathbf{p}^{k}-\mathbf{p}^{k} \mathbf{D}^{k} \mathbf{D}^{k T}\right\|_{F}^{2} \quad \text { s.t. } \mathbf{D}^{k T} \mathbf{D}^{k}=\mathbf{I} \\
\text { Where } \hat{\mathbf{D}}^{k}=\left[\begin{array}{c}
\hat{\mathbf{D}}_{h}^{k} \\
\hat{\mathbf{D}}_{l}^{k}
\end{array}\right], \quad \mathbf{p}^{k}=\left[\begin{array}{c}
\mathbf{P}_{h}^{k} \\
\mathbf{P}_{l}^{k}
\end{array}\right], \quad k=1, \ldots, K .
\end{gathered}
$$

Denote by $\boldsymbol{\Omega}^{k}$ the co-variance matrix of sub-dataset $\mathbf{p}^{k}$. By applying PCA to $\boldsymbol{\Omega}^{k}$, an orthogonal transformation matrix can be obtained. We set the matrix as the sub-dictionary $\hat{\mathbf{D}}^{k}$.

\subsection{Sparse Coefficients Estimation}

For a given LR MS image $\mathbf{Y}$ corresponding to the HR PAN image $\mathbf{X}_{h}$ with $\mathrm{N}$ channels, $\mathbf{Y}$ are tiled into partially overlapping patches $y_{i}$, where $\varphi i$ stands for the $i$-th channel and $i=1, \ldots, N$. As mentioned above, we have learned sub-dictionary pairs $\left\{\hat{\mathbf{D}}_{h}^{k}, \hat{\mathbf{D}}_{l}^{k}\right\}$ and obtained the centroid $\mu_{k}$ of each cluster. With regard to the observed LR MS image, we also extract the features $\mathbf{Y}$ using four 1-D filters in Eq. (1). Then, we select the corresponding sub-dictionary based on the minimum distance between $\hat{y}_{i}$ and the centroid $\mu_{k}$ by the following formula:

$$
k_{i}=\arg \min \left\|\hat{y}_{i}-\mu_{k}\right\|_{2}
$$

By using Eq. (4), we can find the most similar patches $\hat{y}_{i}^{k}$ in the $k_{i}$-th cluster, which has sparse representation over the $k_{i}$-th sub-dictionary.

This step attempts to represent each LR MS patch $\hat{y}_{i}^{k}$ as a linear combination of the atoms of the dictionary $\hat{\mathbf{D}}_{l}^{k}$ with a coefficient vector denoted by $\alpha^{k}$. We argue that it is very likely that the "best" solution is the one employing the least number of the atoms. Therefore, for each LR MS patch $\hat{y}_{i}^{k}$, a sparse coefficient vector $\alpha^{k}$ is estimated by the following formula: 


$$
\hat{\alpha}^{k}=\arg \min _{\alpha^{k}}\left\|\hat{y}_{i}^{k}-\hat{\mathbf{D}}_{l}^{k} \alpha^{k}\right\|_{2}^{2}, \text { s.t. }\left\|\alpha^{k}\right\|_{0} \leq L
$$

where $L$ denotes the sparsity threshold. Equation (5) can be rewritten as follows:

$$
\hat{\alpha}^{k}=\arg \min _{\alpha^{k}}\left\|\hat{y}_{i}^{k}-\hat{\mathbf{D}}_{l}^{k} \alpha^{k}\right\|_{2}^{2}+\lambda\left\|\alpha^{k}\right\|_{0}
$$

where $\lambda$ is the standard Lagrangian multiplier, balancing the sparsity of the solution and the fidelity of the approximation to $\hat{y}_{i}^{k}$. Due to the orthogonality of $\hat{\mathbf{D}}_{l}^{k}$ and the L0 regularization of $\alpha^{k}$, Eq. (4) can be solved by hard thresholding:

$$
\alpha^{k}=H_{\lambda}\left(\hat{\mathbf{D}}_{l}^{k T} \hat{y}_{l}^{k}\right)
$$

where $H_{\lambda}$ is the threshold operator, i.e., $H_{\lambda}(\Theta)=\left\{\begin{array}{c}\Theta,|\Theta|>\lambda \\ 0,|\Theta| \leq \lambda\end{array}\right.$.

\subsection{HR MS Image Reconstruction}

Due to the fact that the sub-dictionaries are built up from the PAN image observing the same area and acquired at the same time as the multispectral channels, the LR MS image patches and their corresponding HR patches to be reconstructed are expected to have a sparse representation in this coupled unitary HR/LR dictionary pair. Furthermore, it is reasonable to assume that there exists a hidden mapping where the different resolutions images can be converted to each other. To keep the assumption simple, many literatures $[7,8,15]$ supposed that the representation coefficients of the image pair should be equal. In this paper, we also assume that each of the HR image patches share the same sparse coefficients as the corresponding LR image patch in the dictionary pair, i.e., the coefficients of the corresponding $k_{i}$-th cluster HR MS image patches $x_{i}^{k}$ in $\hat{\mathbf{D}}_{h}^{k}$ are identical to the coefficients of $\hat{y}_{i}^{k}$ in $\hat{\mathbf{D}}_{l}^{k}$. In the end, the final sharpened multispectral image patches $\hat{x}_{i}^{k}$ are reconstructed by

$$
\hat{x}_{i}^{k}=\mathbf{D}_{h}^{k} \alpha^{k}
$$

The tiling and summation of all patches in all individual channels finally give the desired pan-sharpened image $\hat{\mathbf{X}}$.

\section{Experiments Results}

\subsection{Experiments on Worldview-2 Data Set}

In this section, we evaluate our proposed method on Worldview-2 sensing image and both the sizes of PAN and up-sampled LR MS images are $512 \times 512$. The MS images 
have four bands, i.e., RGB and near infrared (NIR). In the sub-dictionaries learning stage, we randomly sample 100000 image patch pairs $\left\{\mathbf{p}_{h}, \mathbf{p}_{l}\right\}$ with the same size of $7 \times 7$. In addition, we set the threshold $\Delta=10$ to exclude the smooth patches and set the Lagrangian multiplier $\lambda=0.15$. As a clustering-based method, an important issue is the selection of the number of classes. A too large or a too small number of the classes will make the learned sub-dictionaries less reliable. Based on the above considerations, we propose the following simple method to find a good number of classes: we first partition the training dataset into 200 clusters, and merge those classes that contain very few image patches (i.e., less than 300 patches) to their nearest neighboring classes. The original dual images are first downsampled to a lower resolution with bicubic interpolation (the downsampling factor is 1/2) and then fused back to the original resolution. Therefore, the HR MS image is available and the quality of the pan-sharpened images can be measured using full-reference image quality metrics.

We compare the performance with other existing fusion methods, namely, the adaptive IHS (AIHS) [3], the PCA method [4], Wavelet-based image fusion method [6], and SparseFI method [8]. Figures 2(a) and (b) show a Worldview-2 LR MS image (visualized using the color composite of red, green, and blue bands) and a PAN image, respectively. Figure 2(h) shows the reference HR MS image. Figures 2(c) and (g) show the pan-sharpened HR MS images obtained by five different methods.

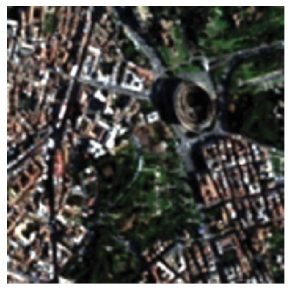

(a)

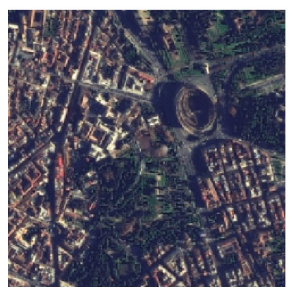

(e)

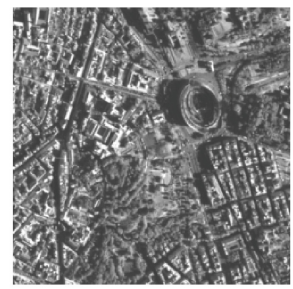

(b)

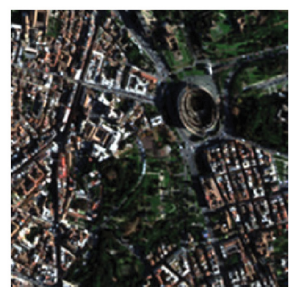

(f)

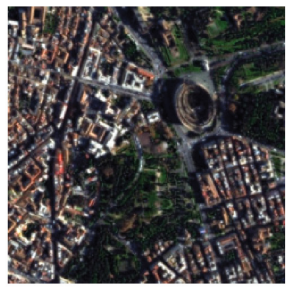

(c)

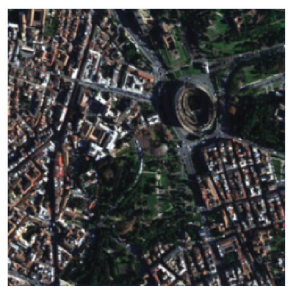

(g)

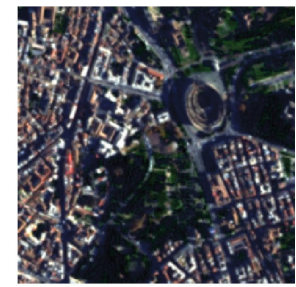

(d)

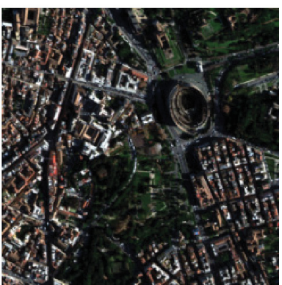

(h)

Fig. 2. Worldview-2 images and experimental results by different methods. (a) Resampled LR MS image. (b) PAN image. (c) AIHS method. (d) PCA method. (e) Wavelet-based method. (f) SparseFI method. (g) Proposed method. (h) Reference MS image.

Obviously, Fig. 2(c) produces halo artifacts around edges compared with the reference HR MS image. Figure 2(d) has poor visual perception due to its serious spatial distortions and spectral distortions. It can be seen that Fig. 2(e) generates good contrast, but it has an obvious chromatic aberration compared with the reference MS image, 
which reflects poor performance in preserving the spectral information. Figure 2(f) preserves the spectral properties better than the above methods, but it shows poor spatial results in details. By contrast, the proposed method in Fig. 2(g) can produce a pan-sharpened image looking very similar to the reference HR MS image. It can not only preserve the spectral information, but also show much finer details than all the methods.

In this paper, five widely used qualitative assessments, i.e., correlation coefficient (CC), root-mean squared error (RMSE), spectral angle mapper (SAM), error relative dimensionless global error in synthesis (ERGAS) and universal image quality indexes (Q4) are adopted. The objective qualitative assessments of fused images in Fig. 2 are shown in Table 1, in which the best results for each criterion are labeled in bold. It can be seen that the proposed method shows the best pan-sharpening performance in terms of all image quality indexes.

Table 1. Comparison of the proposed method with other methods on Worldview-2 data set

\begin{tabular}{l|l|l|l|l|l}
\hline Method & AIHS & PCA & Wavelet & SparseFI & Proposed \\
\hline CC & 0.9291 & 0.8904 & 0.9184 & 0.9369 & $\mathbf{0 . 9 4 6 9}$ \\
\hline ERGAS & 4.9479 & 7.7581 & 5.1201 & 4.7095 & $\mathbf{4 . 0 6 3 3}$ \\
\hline RMSE & 0.0643 & 0.1027 & 0.0674 & 0.0613 & $\mathbf{0 . 0 5 1 9}$ \\
\hline SAM & 5.3603 & 8.6601 & 7.6128 & 4.4015 & $\mathbf{4 . 2 4 7 0}$ \\
\hline Q4 & 0.7907 & 0.6221 & 0.7134 & 0.8236 & $\mathbf{0 . 8 4 9 8}$ \\
\hline
\end{tabular}

\subsection{Experiments on Quickbird Data Set}

To further verify the effectiveness of the proposed method, we move on to conduct the experiments on Quickbird data set. The scene captures over North Island, New Zealand, in Aug. 2012. This dataset consists of a $512 \times 512$ PAN image and the corresponding LR MS image with the size of $128 \times 128$. The MS image has four bands, i.e., RGB and near infrared (NIR). In this experiment, the size of PAN image, the number of image patch pairs, and other parameters are the same as those on Worldview-2 data set.

Figure 3(a) shows a resampled color LR MS image. Figure 3(b) gives the corresponding HR PAN image. The reference HR MS image is shown in Fig. 3(h). As shown in the Fig. 3(c)-(g), they are reconstructed by AIHS method, PCA method, WT method, sparseFI method and the proposed method, respectively. Figures 3(c) and (d) produce serious spatial distortions. Figure 3(e) preserves good spatial information, but suffers from significant spectral distortions. Figure 3(f) gains better performance on a tradeoff between the spatial and spectral information than Fig. 3(c) and (d), but it has great difference in spatial details compared with Fig. 3(h). On the whole, the proposed method shown in Fig. 3(g) outperforms other pan-sharpening methods, as well as the conclusions in Fig. 2.

The objective qualitative assessments of fused images in Fig. 3 are shown in Table 2, in which the best results for each criterion are labeled in bold. Similar to the Table 1, the proposed method performs best in all quality indexes. Therefore, the 


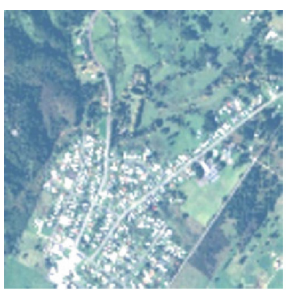

(a)

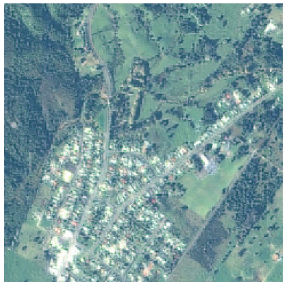

(e)

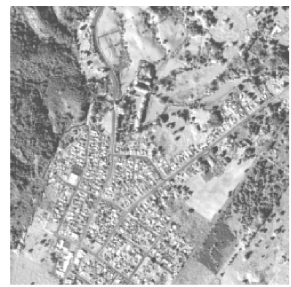

(b)

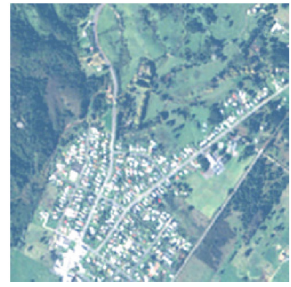

(f)

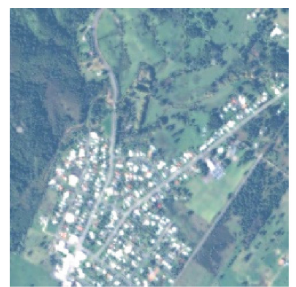

(c)

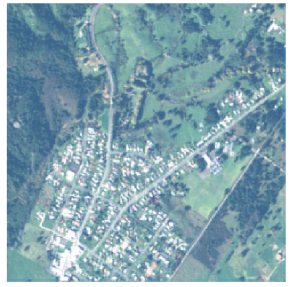

(g)

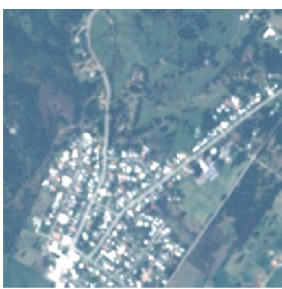

(d)

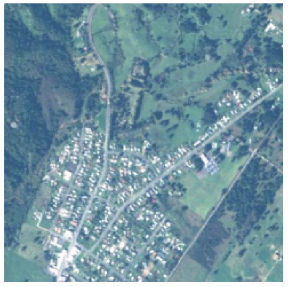

(h)

Fig. 3. Quickbird images and experimental results by different methods. (a) Resampled LR MS image. (b) PAN image. (c) AIHS method. (d) PCA method. (e) Wavelet-based method. (f) SparseFI method. (g) Proposed method. (h) Reference MS image.

Table 2. Comparison of the proposed method with other methods on Quickbird data set

\begin{tabular}{l|l|l|l|l|l}
\hline Method & AIHS & PCA & Wavelet & SparseFI & Proposed \\
\hline CC & 0.9056 & 0.8510 & 0.8825 & 0.9065 & $\mathbf{0 . 9 1 1 2}$ \\
\hline ERGAS & 5.0827 & 6.4203 & 5.3053 & 4.6749 & $\mathbf{3 . 6 9 4 4}$ \\
\hline RMSE & 0.0412 & 0.0553 & 0.0387 & 0.0365 & $\mathbf{0 . 0 2 8 6}$ \\
\hline SAM & 5.9755 & 7.6028 & 8.1403 & 4.4015 & $\mathbf{4 . 0 2 3 2}$ \\
\hline Q4 & 0.6808 & 0.6496 & 0.5790 & 0.6861 & $\mathbf{0 . 7 0 6 1}$ \\
\hline
\end{tabular}

proposed method produces the best fused results and performs the robustness for both the Worldview-2 and Quickbird data sets.

\section{Conclusion}

In this paper, we propose a new pan-sharpening method by coupled dictionary learning and clustered sparse representation for remote sensing image fusion problem. Sub-dictionaries learning overcome the drawback with those atoms which irrelevant to the given local patch in a universal dictionary. We use K-means algorithm and PCA technique to acquire the clusters and each corresponding sub-dictionary. After coupled unitary sub-dictionaries training, the proposed method will be able to reconstruct the HR MS image from the observed LR MS image, since we assume that each of the HR image patches share the same sparse coefficients as the corresponding LR image patch in the coupled HR/LR dictionary pair. Experimental results have demonstrated the 
proposed method can achieve better performance outperforms state-of-the-art methods in terms of visual perception and numerical measures.

Acknowledgement. The authors would like to thank the anonymous reviewers for their careful reading and useful comments on this paper. This research is supported by the Fundamental Research Funds for the Central Universities (30915012204), the National Nature Science Foundation of China (61171165, 11431015), National Scientific Equipment Developing Project of China (2012YQ050250) and Six Top Talents Project of Jiangsu Province (2012DZXX-036).

\section{References}

1. Thomas, C., Ranchin, T., Wald, L.: Synthesis of multispectral images to high spatial resolution: a critical review of fusion methods based on remote sensing physics. IEEE Trans. Geosci. Remote Sens. 46(5), 1301-1312 (2008)

2. Carper, W.J.: The use of intensity-hue-saturation transformations for merging SPOT panchromatic and multispectral image data. Photogramm. Eng. Remote Sens. 56(4), 457467 (1990)

3. Rahmani, S., Strait, M., Merkurjev, D.: An adaptive IHS pan-sharpening method. IEEE Geosci. Remote Sens. Lett. 7(4), 746-775 (2010)

4. Chen, M., Xuan, J.H., Li, D.R.: Image fusion algorithm based on independent component analysis. Opto-Electron. Eng. 34(11), 82-84 (2007)

5. Zhang, Y.: Problems in the fusion of commercial high-resolution satelitte as well as Landsat 7 Images and Initial Solutions. Int. Arch. Photogrammetry Remote Sens. Spat. Inf. Sci. 34 (4), 587-592 (2002)

6. Otazu, X., González-Audícana, M., Fors, O.: Introduction of sensor spectral response into image fusion methods. IEEE Trans. Geosci. Remote Sens. 43(10), 2376-2385 (2005)

7. Li, S., Yang, B.: A new pan-sharpening method using a compressed sensing technique. IEEE Trans. Geosci. Remote Sens. 49(2), 738-746 (2011)

8. Zhu, X.X., Bamler, R.: A sparse image fusion algorithm with application to pan-sharpening. IEEE Trans. Geosci. Remote Sens. 51(5), 2827-2836 (2013)

9. Aharon, M., Elad, M., Bruckstein, A.: K-SVD: an algorithm for designing overcomplete dictionaries for sparse representation. IEEE Trans. Sign. Process. 54(11), 4311-4322 (2006)

10. Elad, M., Yavneh, I.: A plurality of sparse representations is better than the sparsest one alone. IEEE Trans. Inf. Theor. 55(10), 4701-4714 (2009)

11. Protter, M., Yavneh, I., Elad, M.: Closed-form MMSE estimation for signal denoising under sparse representation modeling over a unitary dictionary. IEEE Trans. Sign. Process. 58(7), 3471-3484 (2010)

12. Zeyde, R., Elad, M., Protter, M: On single image scale-up using sparse-representations. In: Proceedings of the 7th International Conference on Curves and Surfaces, pp. 711-730 (2010)

13. Freeman, W.T., Pasztor, E.C., Carmichael, O.T.: Learning low-level vision. Int. J. Comput. Vision 40(1), 25-47 (2000)

14. Sun, J., Zheng, N.N., Tao, H., Shum, H.Y.: Image hallucination with primal sketch priors. Computer Society Conference on Computer Vision and Pattern Recognition, 2, pp. II-729 (2003)

15. Yang, J., Wright, J., Huang, T.S.: Image super-resolution via sparse representation. IEEE Trans. Image Process. 19(11), 2861-2873 (2010) 